\title{
Immunity to viruses
}

The traditional view of immunity to viruses has heavily emphasised the role of serum and secretory antibody and neglected the role of cellular immunity and non-immune host defences such as interferons. ${ }^{1}$ Boys with isolated antibody deficiency ( $\mathrm{X}$ linked hypogammaglobulinaemia) and normal or near normal cellular immunity, however, recover normally from most virus infections with the exception of enteroviruses (echo and coxsackieviruses can cause persistent, often fatal, central nervous system and muscle infection and there is an increased risk of paralytic poliomyelitis). In contrast, children with isolated deficiencies of cellular immunity ${ }^{2}$ and those with acquired deficiencies of cellular immunity but normal antibody production, such as children on cytotoxic or immunosuppressive treatment, develop severe and often fatal infections with measles, chickenpox, respiratory syncytial, influenza, and parainfluenza viruses. $^{3}$

\section{Role of antibody}

Antibody is important in preventing viral infection and reinfection but far less important in recovery from acute infection. Antibodies are produced by B lymphocytes or B cells, which have been activated to plasma cells. Such B cell activation requires assistance from 'helper' cells called helper $T$ lymphocytes or $T$ helper $\left(T_{H}\right)$ cells. Acute infection results in serum IgM antibody production followed by serum IgG. Secretory $\operatorname{IgA}$ is produced locally in response to infection, although IgA production is poor in the neonatal period and early infancy. These antibodies are probably of only minor importance in recovery from viral infection, as antibody deficient individuals can recover normally. For example, nasal secretory antibody against respiratory viruses generally appears too late to limit viral replication. ${ }^{3-6}$

Antibody does, however, seem to be important in preventing infection or at least decreasing the severity. Transplacental IgG protects neonates and infants against virus infection. Passive administration of hyperimmune gammaglobulin against varicella zoster virus or measles virus decreases the severity if not the incidence of infection in newborns or immunocompromised subjects. ${ }^{7}$ The presence of pre-existing specific nasal IgA correlates better than serum antibody with protection against respiratory viral infection. ${ }^{57}$ IgA deficiency is usually found coincidentally in normals, but may also be associated with recurrent infections of the respiratory and gastrointestinal tracts. Deficiencies of IgG subclasses, particularly $\operatorname{IgG}_{2}$, are also associated with recurrent respiratory tract infections.

\section{Cellular immunity}

\section{(1) T CELLS AND ANTIGEN PROCESSING}

Thymus derived or $T$ lymphocytes ( $T$ cells) are responsible for cellular or cell mediated immunity. They will only recognise foreign antigens, however, which have been 'processed' and presented on the surface of antigen presenting cells. Almost any cell can act as an antigen presenting cell, but macrophages and monocytes are the most extensively studied. Macrophages recognise foreign antigens, ingest them and 'process' them. T cells only recognise antigens presented by cells which share the same HLA type. It is now known that macrophages present processed antigen on the surface of the cell, in intimate conjunction with one of their major histocompatibility complex, also called HLA antigens. Thus the T cell response is said to be major histocompatibility complex restricted.

In elegant studies on the recognition by $T$ cells of influenza virus infected cells, Townsend and Skehel have shown that it is not the surface proteins of influenza virus that are primarily recognised but the nucleoprotein. ${ }^{8}$ This seems intuitively wrong as nucleoprotein would be expected to be expressed in the nucleus of infected cells, not on the cell surface. Townsend went on to show, however, that the T cell is actually recognising short peptides of denatured influenza virus nucleoprotein, only about 14 amino acids long, expressed on the cell surface. ${ }^{9}$ Similar recognition of viral peptides by cytotoxic $\mathrm{T}$ lymphocytes has since been shown for human immunodeficiency virus. ${ }^{10}$ Thus it seems that antigen presenting cells can break down viral proteins and express one or more of the resulting peptides on their surface for $T$ cells to recognise. It now appears that the peptide lies in a groove in the major histocompatibility complex molecule on the antigen presenting cell's surface where it can be recognised by $T$ cells ${ }^{11}$; if a single amino acid in the peptide is changed this may alter its shape so it will not fit in the groove.

The $T$ cell recognises the viral peptide through a 
specific $\mathrm{T}$ cell receptor, which has constant and variable regions that can be thought of as analogous to immunoglobulin (fig 1). It becomes irreversibly 'committed', discards the DNA for other receptors, and starts to proliferate. It then can mature either into a memory cell, which becomes quiescent but recognises the same antigen if re-exposed, or into an effector cell. These effector cells can either destroy infected cells (cytotoxic $T$ cells) or help B cells to produce antibody ( $\mathrm{T}$ helper cells). $\mathrm{T}$ cells also release soluble proteins, lymphokines, such as interferon gamma (see below), which recruit inflammatory cells and thus limit the spread of infection.

Cytotoxic $\mathrm{T}$ cells seem to be important in protection against influenza virus infection ${ }^{12}$ and in recovery from bronchiolitis caused by respiratory syncytial virus. ${ }^{13}$ However, they may also contribute to disease. Cytotoxic $\mathrm{T}$ lymphocytes are found in the blood and cerebrospinal fluid in mumps meningitis, and a pathogenic role has been suggested. ${ }^{14}$

\section{(2) NATURAL KILLER CELLS}

Natural killer cells resemble large granular lymphocytes morphologically, but probably come from a lineage distinct from $B$ and $T$ cells. They can kill virus infected cells, as well as tumour cells and some micro-organisms. This is not an immune mechanism as it occurs spontaneously without prior exposure, and does not require antigen processing or major

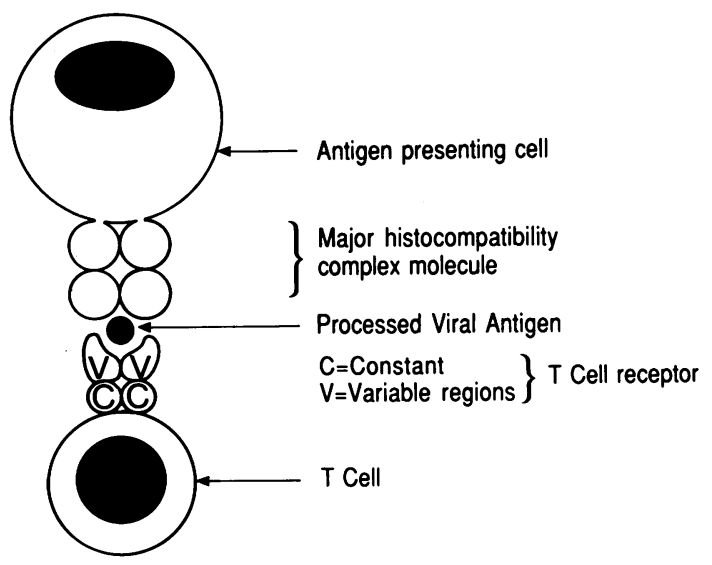

Fig 1 Diagrammatic representation of the recognition by a cytotoxic $T$ cell of viral prptide presented by an antigen presenting cell in association with a major histocompatibility complex molecule. (The illustration is from Isaacs D, Moxon R, Neonatal Infections; this is to be published by Butterworths in late 1990.) histocompatibility complex recognition. Natural killer cells are stimulated by both interferon alfa and interferon gamma (see below) and become more active. The importance of natural killer cell activity in different viral infections varies: natural killer cells seem to be particularly important in limiting infections due to herpes viruses. ${ }^{15}$

\section{(3) ANTIBODY DEPENDENT CELLULAR CYTOTOXICITY} The role of antibody dependent cellular cytotoxicity in viral infections is unclear. The responsible cells, predominantly lymphocytes, recognise viral antigen bound to antibody and will then kill virus infected cells. Like natural killer cells they are activated by interferon gamma.

\section{Interferons}

There are three types of interferon: alfa, beta, and gamma. ${ }^{16}$ Interferon alfa can be produced by almost any nucleated cell in the body and interferon beta by fibroblasts and epithelial cells in response to various stimuli, particularly virus infection. They then act on neighbouring cells, stimulating them to produce various antiviral proteins that render them resistant to viral infection. This is not an immune mechanism: interferons alfa and beta can be produced in response to a wide range of viruses and also protect against a wide range of viruses.

Interferon alfa is produced locally in most respiratory virus infections before antibody can be detected and is probably one of the most important mechanisms of recovery from such infections. In general interferon alfa is not produced by the body in the absence of infection, although bronchoalveolar lavage of healthy volunteers has shown high resting lung concentrations of interferon alfa, a finding of uncertain significance. ${ }^{17}$ If mice are pretreated with anti-interferon alfa antiserum they become far more susceptible to infection with herpes simplex virus, encephalomyocarditis virus and other viruses, though not to influenza virus. ${ }^{18} 19$ Interferon alfa does not appear to be important in recovery from respiratory syncytial virus infection. ${ }^{20}$ Children with defective production of interferon alfa, apparently due to delayed maturation of production, however, have an increased incidence of respiratory viral infections with wheeze. ${ }^{21}$

Interferon gamma, or immune interferon, in contrast to interferon alfa, is produced by $T$ cells on re-exposure to an antigen to which they have already been sensitised and is thus an immune mechanism. Although interferon gamma has antiviral properties, it appears to have a more important role as a modulator of the immune response. Macrophages and monocytes are activated by interferon 


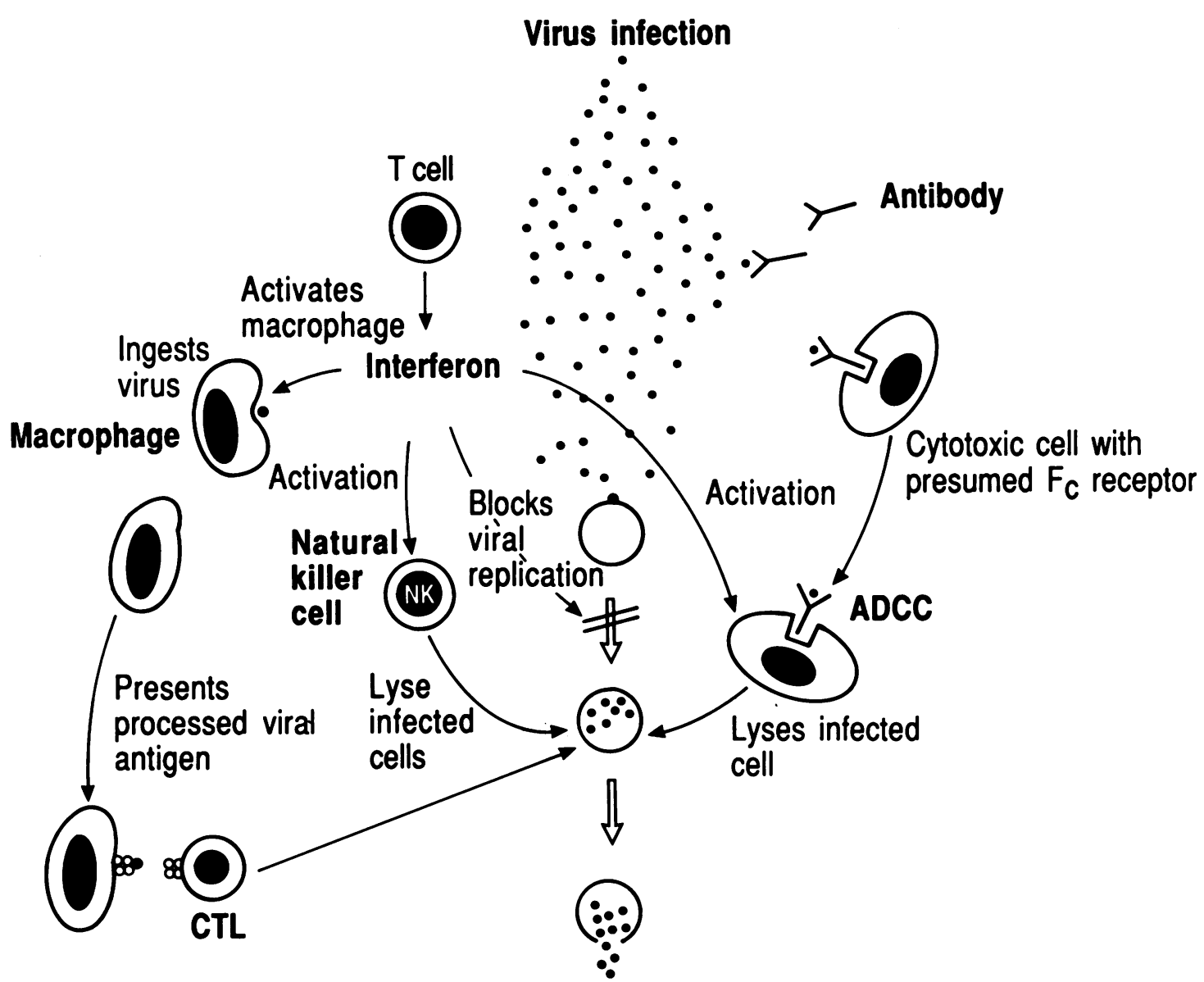

Fig 2 Interaction of various host defence mechanisms in recovery from viral infection. ADCC=antibody dependent cellular cytotoxicity, $C T L=$ cytotoxic T lymphocytes. (The illustration is from Isaacs D, Moxon R, Neonatal Infections; this is to be published by Butterworths in late 1990.)

gamma, thus improving their ability to process and present antigen, while natural killer cells and the cells responsible for antibody dependent cellular cytotoxicity are activated to increase lysis of virus infected cells. ${ }^{16}$

\section{Neutrophils}

There is evidence that neutrophils are important in some virus infections, although neutropenia is more often found as a consequence of virus infections than it is recognised as a cause of them. Nevertheless, a neutrophil leucocytosis is seen early in experimental infections with rhinovirus, influenza, and coxsackieviruses and late in adeno- virus infections. Some viruses-for example, herpes simplex and mumps-generate chemotaxins, and neutrophils can adhere to virus infected cells in vitro. Many different viruses have been identified within neutrophils and can activate neutrophil oxidative metabolism and intracellular killing. ${ }^{22}$

\section{Conclusion}

The defence mechanisms that have been described are of varying importance in different virus infections. It is becoming increasingly clear that the different mechanisms can interact with each other to amplify the immune response to virus infection. The 
release of lymphokines from activated $T$ cells will activate a number of different cells to improve their ability to limit the spread of infection (fig 2).

I should like to express my gratitude to the Wellcome Trust for their support, and to Andrew McMichael, Charles Bangham, Alain Townsend, Frances Gotch, and others in the Nuffield Department of Medicine who have stimulated my interest and tolerated my ignorance; I also thank Miss Gail Davies for typing the text.

\section{References}

1 Ogra PL, Morag A, Ogra SS, Beutner KR. Host defense mechanisms in viral respiratory infections. Pediatr Res 1977;11:231-3.

${ }^{2}$ Fishaut M, Tubergen D, McIntosh K. Cellular response to respiratory viruses with particular reference to children with disorders of cell-mediated immunity. Pediatrics 1980;96:179-86.

3 Alford RH, Rossen RD, Butler WT, Kasel JA. Neutralizing and haemagglutination-inhibiting activity of nasal secretions following experimental human infection with $\mathrm{A} 2$ influenza virus. J Immunol 1967;98:724-31.

4 Butler WT, Waldman TA, Rossen RD, Douglas RG, Couch RB. Changes in IgA and IgG concentrations in nasal secretions prior to the appearance of antibody during viral respiratory infections in man. J Immunol 1970;105:584-91.

5 Cate TR, Rossen RD, Douglas RG, Butler WT, Couch RB. The role of nasal secretion and serum antibody in the rhinovirus common cold. Am J Epidemiol 1966;84:352-63.

6 Rossen RD, Butler WT, Cate TR, Szwed CF, Cough RB. Protein composition of nasal secretion during respiratory virus infection. Proc Soc Exp Biol Med 1965;119:1169-76.

7 Rossen RD, Butler WT, Waldman RH, et al. The proteins in nasal secretion. II. A longitudinal study of IgA and neutralising antibody levels in nasal washing from men infected with influenza virus. JAMA 1970;211:1157-61.

8 Townsend ARM, Skehel JJ. The influenza A virus nucleoprotein gene controls the induction of both subtype specific and cross-reactive cytotoxic T-cells. J Exp Med 1984;160:552-63.

9 Townsend ARM, Rothbard J, Gotch FM, Bahadur G, Wraith $D$, McMichael AJ. The epitopes of influenza nucleoprotein recognized by cytotoxic $\mathrm{T}$ lymphocytes can be defined with short synthetic peptides. Cell 1986;44:959-68.

${ }^{10}$ Nixon DF, Townsend ARM, Elvin JG, Rizza CR, Gallway J,
McMichael AJ. HIV-1 GAG specific cytotoxic lymphocytes defined with recombinant vaccinia virus and synthetic peptides. Nature 1988;336:484-7.

1 Bjorkman PJ, Saper MA, Samraoui B, Bennett WS, Strominger JL, Wiley DC. Structure of the human class I histocompatibility antigen, HLA-A2. Nature 1987;329:506-12.

12 McMichael AJ, Gotch FM, Noble GR, Beare PAS. Cytotoxic Tcell immunity to influenza. N Engl J Med 1983;309:13-7.

13 Isaacs D, Bangham CRM, McMichael AJ. Cell-mediated cytotoxic response to respiratory syncytial virus in infants with bronchiolitis. Lancet 1987;ii:769-71.

14 Kreth HW, Kress L, Ott HF, Eckert G. Demonstration of primary cytotoxic T-cells in venous blood and cerebrospinal fluid of children with mumps meningitis. $J$ Immunol 1982;128:2411-5.

15 Biron CA, Byron KS, Sullivan JL. Severe herpesvirus infections in an adolescent without natural killer cells. $N$ Engl $J$ Med 1989;320:1731-5.

16 Balkwill FR. Interferons. Lancet 1989;i:1060-3.

17 Prior C, Haslam PL. Interferons in lungs. Lancet 1989;i:1333.

18 Gresser I, Tovey MG, Bandu MT, Maury C, Bronty-Boye D. Role of interferon in the pathogenesis of virus diseases in mice as demonstrated by the use of anti-interferon serum. I. Rapid evolution of encephalomyocarditis virus infection. J Exp Med 1976;144:1305-15.

19 Gresser I, Tovey MG, Maury C, Bandu MT. Role of interferon in the pathogenesis of virus diseases in mice as demonstrated by the use of anti-interferon serum. II. Studies with herpes simplex, Moloney sarcoma, vesicular stomatitis, Newcastle disease and influenza viruses. J Exp Med 1976;144:1316-27.

20 Isaacs D. Production of interferon in respiratory syncytial virus bronchiolitis. Arch Dis Child 1989;64:92-5.

21 Isaacs D, Clarke JR, Tyrrell DAJ, Webster ADB, Valman HB. Deficient production of leucocyte interferon (interferon-alpha) in vitro and in vivo in children with recurrent respiratory tract infections. Lancet 1981;ii:950-2.

${ }^{22}$ Faden H, Ogra P. Neutrophils and antiviral defense. Pediatr Infect Dis 1986;5:86-92.

D IsAACs
Infectious Diseases Unit,
Department of Paediatrics,
John Radcliffe Hospital,
Headington,
Oxford OX3 9DU

See related papers on $\mathrm{p} 1656$ and $\mathrm{p} 1661$. 